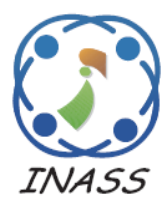

\title{
Low Latency FiWi Enhancement with Tactile Internet on Passive Optical Network System
}

\author{
Jirasak Ponchua $^{1 *} \quad$ Suchada Sitjongsataporn $^{2}$ \\ ${ }^{I}$ The Electrical Engineering Graduate Program, Faculty of Engineering, \\ Mahanakorn University of Technology, Bangkok Thailand 10530 \\ ${ }^{2}$ Department of Electronic Engineering, Mahanakorn Institute of Innovation, Faculty of Engineering, \\ Mahanakorn University of Technology, Bangkok Thailand 10530 \\ * Corresponding author's Email: jirasak_ponchua@hotmail.com
}

\begin{abstract}
Explosive growth and usage of internet while COVID-19 pandemic demands an access technology in our lifestyle that means the Internet of Things (IoT) and Passive Optical Networks (PONs) are increasingly being used in order to support the digital lifestyle. Mobile Internet allows us firstly to exchange the content of information and multimedia while on the move. Then, IoT makes it possible to connect the smart devices with the low latency. PONs have been suggested for providing the ultra-low latency for Quality of Service (QoS) applications such as the Tactile Internet (TI) that is the next evolution to enable real-time control of IoT. A one-millisecond round-trip delay is required to support the tactile internet. As stated on the improving bandwidth allocation for PONs and Passive Optical LAN (POLAN), we propose the FiWi-Dynamic Wavelength and Bandwidth Allocation (F-DWBA) algorithm to reduce the round-trip delay at lower than one-millisecond, which the proposed F-DWBA algorithm based on the PONs and WLAN $802.11 \mathrm{~b}$ should have a maximum performance. The lowest round-trip delay will make the Fiber-Wireless (FiWi) system can work efficiently and support TI applications. Simulation setup are tested on the latency budget of communication for the TI system starting from the Mobile Unit (MU) through the access point (AP) connected to the Optical Network Unit (ONU) including with the Optical Line Terminal (OLT) to the server. Performance analysis is focused on three periods of the latency time at the optical access network, radio interface and MU interface. Simulation results show that the proposed F-DWBA algorithm has a better round-trip delay than the Gigabit-Passive Optical Network (G-PON) standard about 40 percentage and better than the conventional Dynamic Wavelength and Bandwidth Allocation (DWBA) algorithm about 33 percentage at the full working of the tactile internet system.
\end{abstract}

Keywords: Passive optical networks (PONs), Tactile internet (TI), Optical network units (ONU), Fiber-wireless (FiWi), Dynamic wavelength and bandwidth allocation (DWBA), Gigabit-passive optical network (G-PON) standard.

\section{Introduction}

Nowadays, we are increasingly using technology in our daily life. According with the coronavirus disease (covid-19), we have started working from home including with the smart home, smart office and smart factory, which have become more active in the business model. The various electronic devices in the smart home, smart office and smart factory can support the network connections, called Internet of Things (IoT) [1].
IoT is a kind of system linked computer devices, machines and humans such as a wearable heart rate monitor for human, a smart biochip for farm animals and the smart sensors for electrical vehicle while driving through an Internet Protocol (IP) address to transfer data over the network. As stated in the smart home, smart office and smart factory and the satellitebased estimated rainfall system [2], the IoT system is used to collecting data from various smart devices in the end-user area. The data is then used to monitor, control, and transfer them to other devices over the internet [3]. However, many IoT devices have worked wirelessly while connecting to the other 
devices. Most IoT devices consume very small bandwidth. But the rising need for online smart devices that truly become a greater demand for bandwidth as well. The IoT is increasingly being used in industrial systems named "industrial internet of things (IIoT)" [4].

The benefit of IoT is the resource efficiency. If we know the functionality and improve the limitations of each device, we can increase its usability more efficiently. Because IoT devices communicate with each other and perform many tasks. Therefore, it helps people work more easily. Network design and management is an important issue that needs to be developed to support the IoT system.

Meanwhile, a passive optical network (PON) system is a connection between the central office and the end user's home, office and factory through the optical fiber. The use of PONs [5] and passive optical local area network (POLAN) [6] for broadband services have been presented currently. Many broadband providers are already being used these technologies to offer broadband services for consumers. PON technology provides effective high bandwidth management for preventing network congestion, which causes the errors and retransmitting. This makes it particularly effective for applications, which require a stable network. PON is a solution to support IoT and the growth of the improved bandwidth and service quality.

Tactile internet (TI) is an internet network combined on the ultra-low latency with extremely high availability, reliability and security, which is the upcoming wave of innovation [7]. For using the realtime and remote controls, the communications system should be a platform for enabling the remote control and direction of real and virtual objects in the different situations. So, the tactile internet will become the next generation of communication and innovation system in terms of very low latency, high availability, reliability, and security. The tactile internet is a precise function of both human-tomachine and machine-to-machine interactions, where the end-to-end response time is within only one millisecond required for internet applications. That means it will deliver a low latency to build the realtime interactive systems. The latency time of the PON standard system does not fully support the tactile internet applications. Improving the latency time in PON technology to support the ability of the tactile internet is imperative for maximum efficiency.

By combining the capacity of optical fiber networks with the ubiquity and mobility of wireless networks, the Fiber-Wireless (FiWi) broadband access networks [8] is a powerful platform for the support and creation of emerging as well as future unforeseen applications and services. In [9], the researchers have proposed the Dynamic Wavelength and Bandwidth Allocation (DWBA) algorithm for improving the latency time from PON to ONU. The DWBA algorithm can limit the latency time between OLT to ONU by one millisecond, but the actual connection of the IoT system will include the mobile unit (MU) devices. Therefore, if the connection between ONU and MU has high latency. The DWBA algorithm does not fully supports the tactile internet system, which the use of the tactile internet requires the latency time within one millisecond in the system from cloud server to MU.

In this paper, we propose an algorithm that can help to make the connection more efficient. The goal of this paper is to focus on the very low latency of the connection system with ultra-high reliability by using an adaptive algorithm to improve the equipment connectivity. The performance of Fiber-Wireless (FiWi) connection and the end-to-end latency will be analyze for both fixed and mobile devices.

In the section 2, we present the internet of things technology, consist of system functionality, and an example of implementation. Section 3 presents the passive optical networks technology consist of system standard and the use of PONs and POLAN. Section 4 presents the tactile internet consist of the latency time requirements. In the section 5, we analyze the latency time of the PON system including the mobile device. Section 6 presents proposed system. Section 7 presents simulation setup and results, and conclusion in the last section.

\section{Internet of things technology}

Internet of things (IoT) systems that are embedded the sensors, processors and software to exchange the data with the other smart devices. IoT devices will share the sensor data collected by connecting to an IoT gateway. Then, the data will be sent to the cloud system for analysis on the local device. Sometimes, these IoT devices will communicate with other neighboring devices involved to analyze the information received from each other.

Fig. 1 shows an IoT system that consists of three main parts including of collecting data, collating and transferring data, and analyzing data-act [10]. In addition to providing the smart devices to smart homes, the IoT systems are being supported the businesses working by offering insights into operations as Customer Relationship Management 


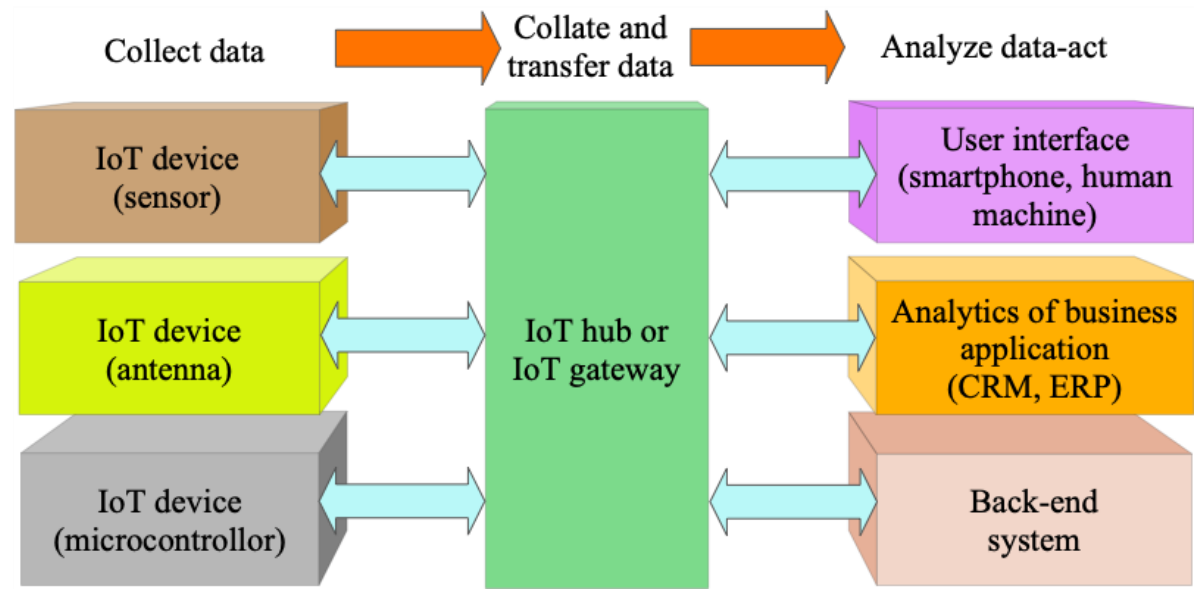

Figure. 1 Example of an IoT system

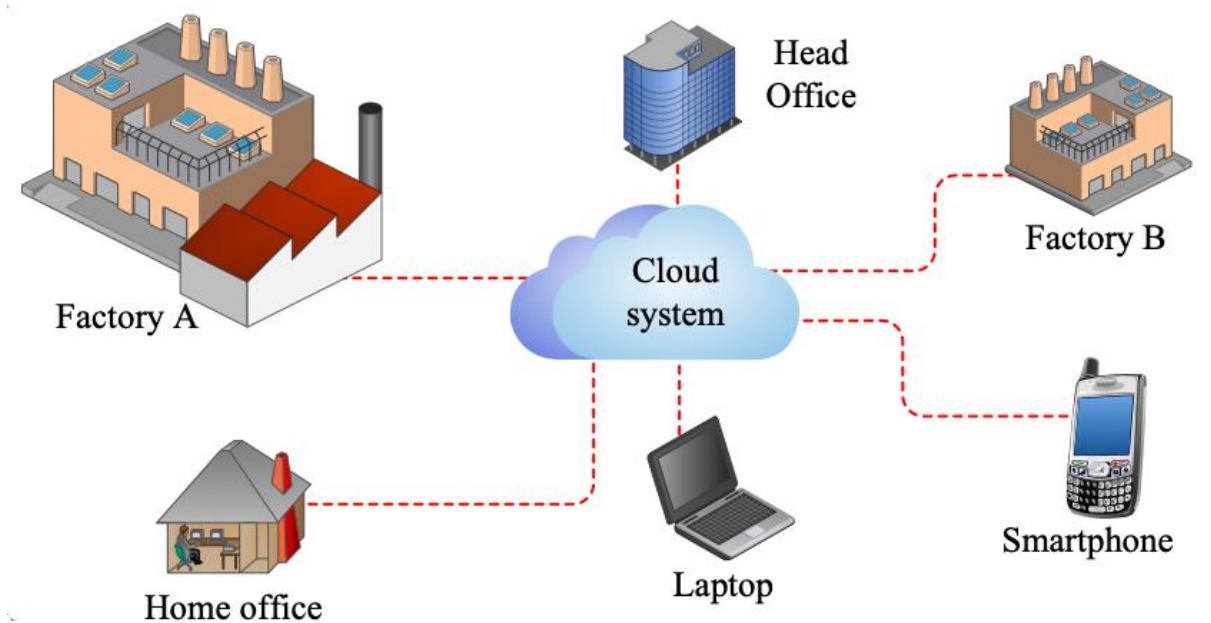

Figure. 2 Example of the industrial internet of things (IIoT)

(CRM), Enterprise resource planning (ERP), supply chain, and logistics. In practice, IoT can be used with sensors connected to a circuit board, which is programmed to measure the range of data collected from sensor devices such as temperature, pressure, vibration, and motion $[11,12]$. So, the IoT sensors enable the seamless data control through automation system that can deliver the actionable insights.

Industry 4.0 is the major changes that occur in how the products are produced and delivered to focus on an industrial automation and flexible factories. In order to compete factories and warehouses, the need of the industrial internet of things (IIoT) and digitization is to become more streamlined and efficient.

For example, the smart factory used the IIoT technology is presented in Fig. 2, which can be connect to the smart factory via the smartphone, programmable through laptop with the cloud storage. For the business industries, IoT can also be the solutions for managing industrial IoT devices. The connected cloud software can be replenished with various resources, allowing the smart control through the different platforms and devices that the connected factory solution can report the key metrics including equipment performance and telemetry.

IIoT solutions can make the machines smarter. The factory will get more efficient with the less wasteful process on production lines, which are more flexible and high productive.

\section{Passive optical networks}

The passive optical networks (PON) is a connection between the central office and the end user's home via optical fiber called "the fiber to the home (FTTH) solution" with the Point-to-Multipoint (P2MP) network. In other architectures, it uses mostly technology called "point-to-point (P2P)" network. In the market, mostly broadband operators are now using the Gigabit Passive Optical Network (GPON) [5] technology to provide the customer service.

GPON is defined the standard by communication and service operations coordination unit as known as the International Telecommunications Union in Telecommunication or ITU-T, named G.984.1 [13]. GPON system is operating at $2488 \mathrm{Mbps}$ downstream 


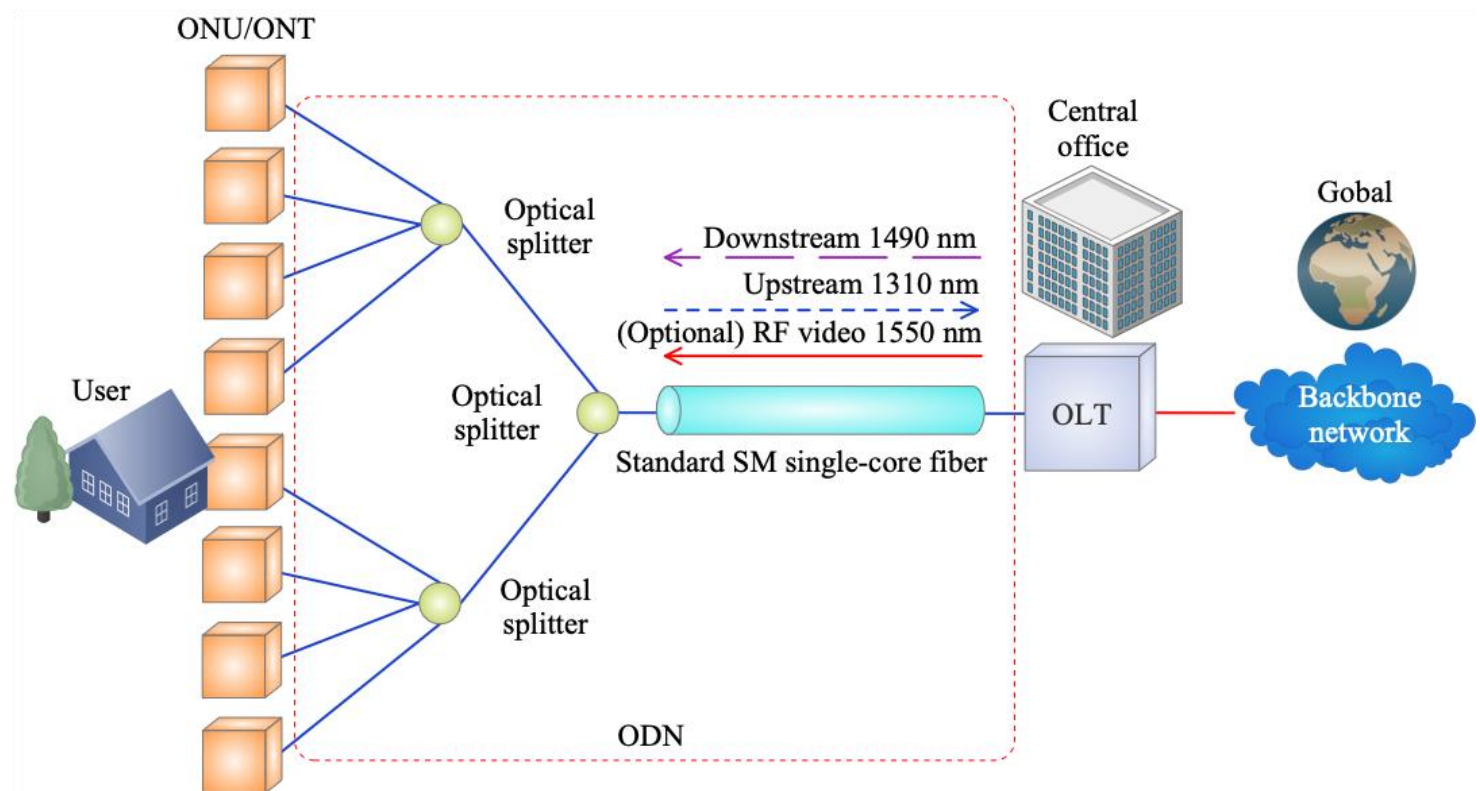

Figure. 3 Topology example of GPON including with the OLT, ODN, and ONU/ONT

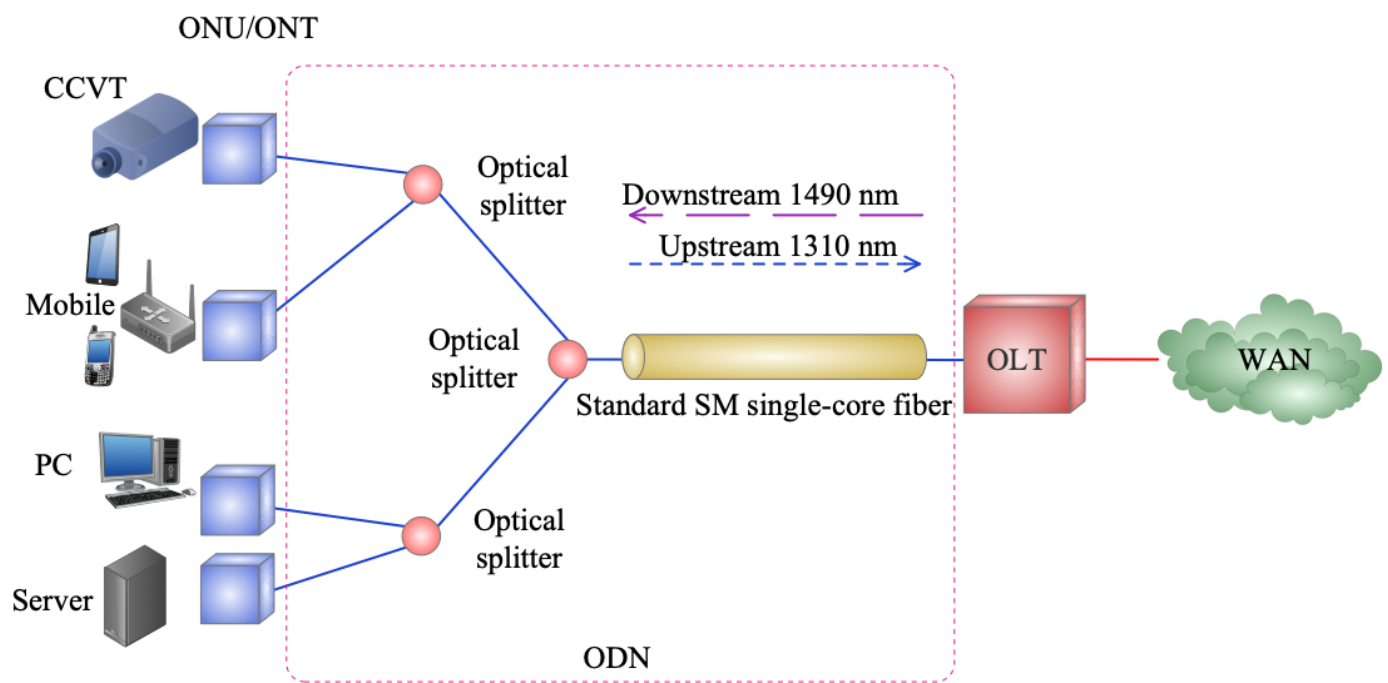

Figure. 4 Topology of passive optical LANs.

rate and 1244 Mbps upstream rate. The Optical Network Units (ONUs) are deployed between zero to $40 \mathrm{~km}$ or the distances with a $40 \mathrm{~km}$ differential distance from the Optical Line Terminal (OLT). GPON consists of three main parts including with Optical Line Terminal (OLT), Optical Distribution Network (ODN) and Optical Network Unit / Terminal (ONU / ONT) as shown in Fig. 3.

The topology of GPON system is presented in Fig. 3. An OLT is a device located in a central office (CO), while an ODN network operates as a passive optical network (PON). This means that there is no a power supply required for operation between the beginning and end of the distribution network. The ONU / ONT is located at the end-user location. The operation wavelength for downstream transmission applications is of $1490 \mathrm{~nm}$. The transmission of data from the ONU/ONT is used at $1310 \mathrm{~nm}$. For example, if there is a video signal in the radio frequency (RF) video format used $1550 \mathrm{~nm}$, the GPON technology can support the IoT operations with an appreciate network [14]. But there will be a problem in bandwidth management and delay time while processing.

The passive optical local area network (POLAN) [15] is applied for point-to-multipoint system and to build on the foundation of PON system. The indoor network infrastructure with the optical splitters is used to deliver data from a single point to multiple users. Although, the POLAN does not occur as long as PON, but it is considered as the next generation of infrastructure to replace the traditional copper-based LANs. 


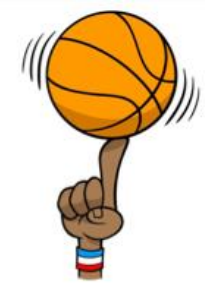

$1 \mathrm{~ms}$

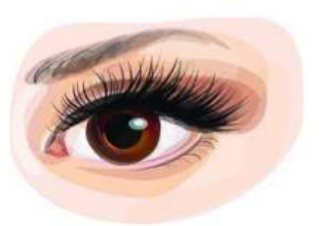

$10 \mathrm{~ms}$

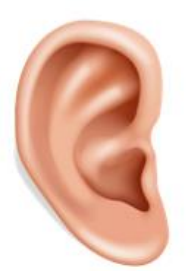

$100 \mathrm{~ms}$

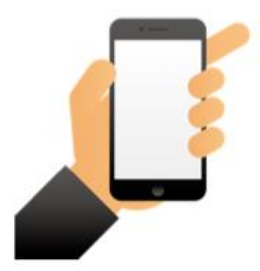

$1 \mathrm{~s}$

Figure. 5 Typical human reaction time

Local area network (LAN) is becoming a network bandwidth bottleneck as the number of end-users in LAN increases as well as a higher demand per enduser data bandwidth. While Category 6 cable (CAT6) at $10 \mathrm{Gbps}$ can be reached within the distance of 55 meters. Even for Category 8 cable (CAT8), the new technology of the copper cable family is of $10 \mathrm{Gbps}$, $25 \mathrm{Gbps}$, and 40Gbps, which are limited within the range up to 30 meters. GPON has many advantages more LAN technology, such as the higher capacities, lower repair rates, and energy savings. As 5G and WiFi-6 system enter to the market, the usage of IoT applications will increase dramatically. Enterprise networks will need to connect many devices in the larger locations with more bandwidth need.

Fig. 4 shows the topology of POLAN by replacing copper cables with single-mode fiber optic cables [15]. It becomes an all-fiber connection with less copper wires connected to the terminal equipment. The OLT applied in POL involves both the upstream and downstream that obtains data from service providers by connecting them to Wide Area Network (WAN) and the enterprise resource services through the main router, after that the data is sent to ONU/ONT system. On the other hand, the OLT also receives information from ONU/ONT and sends data to the service provider. The number of OLT ports are typically connected at 32 ONTs. A passive optical splitter is a core component in a passive optical LAN. There are typically one or more optic splitters that operate between the OLT and the ONU/ONT. These optical splitters can be placed elsewhere in the center of an optical network to distribute the optical signal to the end-user without the electric current need. The ONU/ONT is an interface that connects the end-users in the local network, where the ONT converts the fiber optic signal into an Ethernet RJ45 interface connected to computers and other devices.

For the Dynamic Wavelength and Bandwidth Allocation (DWBA) [16] algorithm to predict information collected from all ONUs, each ONU has various values of the MU connected. In [14], the authors have presented the DWBA algorithm is based on the knapsack 0/1 problem for bandwidth and wavelength allocation. This algortihm is executed when the central office (CO) receives all report messages. Then it takes over all available data to calculate the proportion of bandwidth each ONU for the upcoming cycle. Thereafter, a report message arrives OLT, then OLT will generate a gate message informing ONU. This message will include with the start time and the allowed bandwidth allocated for the next round.

In [18], the DWBA algorithm is used to allocate wavelength and bandwidth of each ONU in order to support the tactile internet. While the Tactile Internet functionality has expanded to more mobile unit (MU). Their work is therefore not fully mobile unit supported.

\section{The tactile internet}

The Tactile Internet is defined as an internet network that combines with low latency, high availability, reliability, and security [7]. The Tactile Internet will enable humans and machines to interact in real-time, while on the move and within a certain spatial communication range. In principle, the human sense can interact with the various machines and technologies to enable and optimize the interaction delivering from the digital world. The cognitive processes have limits on how instantaneously the human can interact with the environment on experience.

The typical human response time is shown in Fig. 5 , which depends what affects the sensory system and how human prepares for the situation. In the presence of sudden responses, the delay time between the human sensing stimuli and the muscular reaction is at one second. If human prepares in advance, we will use a faster response time. The human hearing response time is about 100 milliseconds, so the modern smartphones are designed to ensure that the sound is transmitted within 100 milliseconds. The typical human visual response time is in the range of 10 milliseconds. For smooth video viewing, the modern television receivers have a minimum picture with the refresh rate of $100 \mathrm{~Hz}$ to support the human reaction, where the maximum response time between 


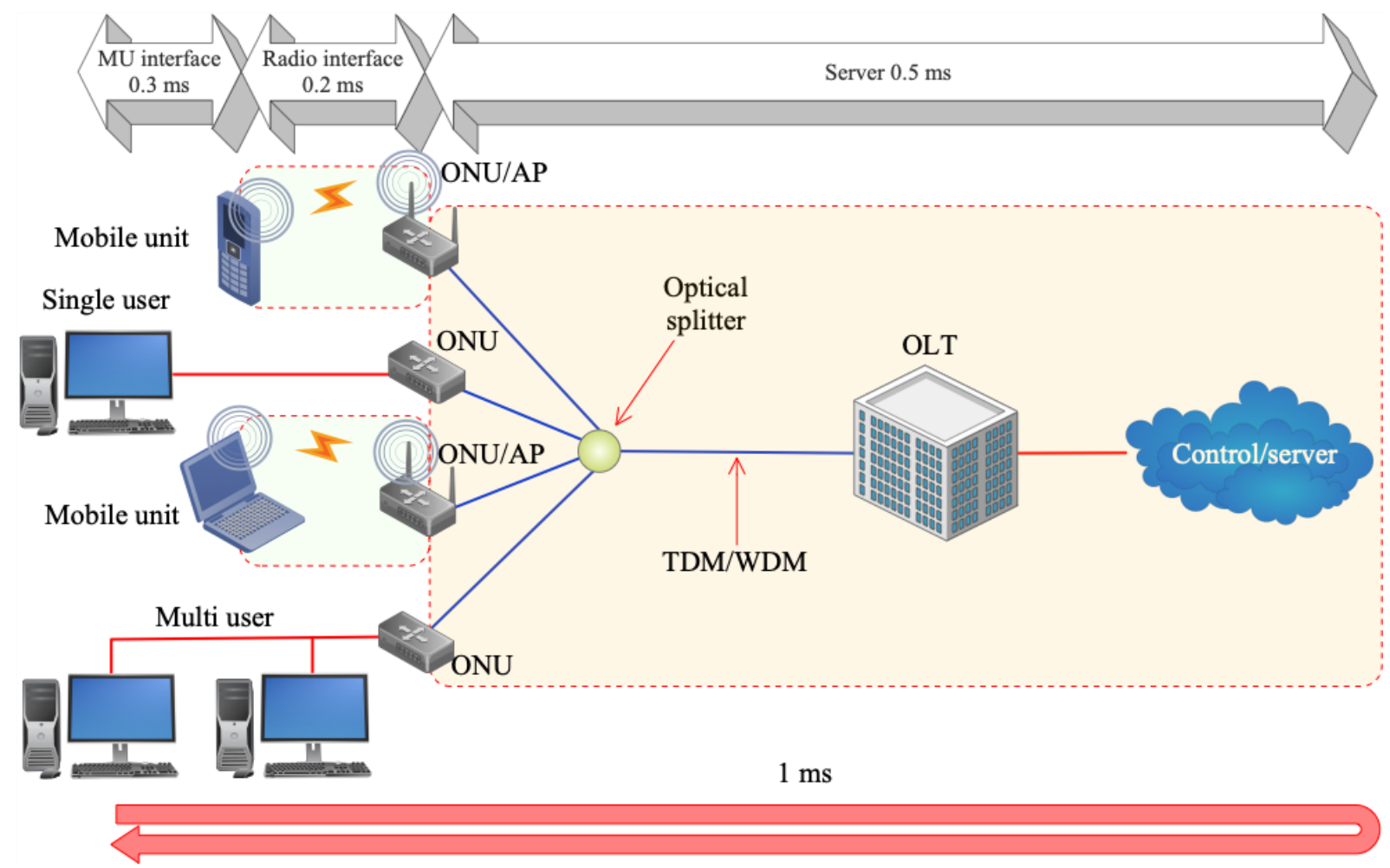

Figure. 6 Latency budget of Tactile Internet system

the shots is 10 milliseconds. In [18], the authors have presented the allocated resources to support the tactile internet system, but there is no experiment in the PON architecture.

The most challenging latency requirement in communication system for tactile internet is of one millisecond for the end-to-end latency. The strict latency requirement for the human body movement is at one millisecond. If the time-lag between the connections with human movement is over than on millisecond, the cyber-sickness may be occurred.

\section{Analysis}

In this section, we analyze the performance of Fiber-Wireless (FiWi) connectivity in terms of latency time. The recent technology to access broadband networks is to use a bi-modal structure, which takes the advantage of the respective strengths of fiber optic and wireless technologies. It is combined to create a FiWi network that supports the user data transmission.

By combining the capabilities of fiber optic networks with the mobility of wireless networks, the FiWi network is a powerful platform for supporting the future applications and services. To achieve the end-to-end one-millisecond latency of tactile internet, the one-millisecond system response is set to be critical.

Fig. 6 shows an exemplary latency budget of communication for the tactile internet, where the sensor in the Mobile Unit (MU) will be measured at the end. The data is pre-processed and provided to the embedded system that controls the air interface, and then passes this data through all protocol layers to the physical layer. Similarly at the receiver, an Access Point (AP) that is connected to the ONU with the data being passed to the control/server, which are taken and made the decision.

In our analysis, there are three periods of latency time defined as follows:

\subsection{Optical access network}

In our access model, we represent the connection from ONU/AP to the cloud server. For GPON fiber networks, the fiber optic cable ITU-T G.652 [17] is commonly used and the fiber cable is a single-mode type. PON standard is to estimate the actual PON fiber distance from the OLT to a particular ONU. The OLT can implement the estimation procedure based on the round-trip measurement, using the value of the ONU actual response time. The estimate of the fibre distance may be obtained according to the following

$$
F D_{i}=\left(R T D_{i}-R T_{i}\right) \cdot 102 \quad,
$$

where $F D_{i}$ is the estimated fibre distance in meters between the OLT and the specified ONU, RTD $i$ is the round-trip delay in microseconds measured by the OLT, $R T_{i}$ is the true ONU response time in microseconds, and the numeric coefficient of 102 
$\mathrm{m} / \mu \mathrm{s}[13]$ is a best fit value reflecting the range of refractive indices that G.652 fibres exhibit in the field. The round trip time (RTT) between OLT and ONU can be defined as:

$$
T_{\text {rtt onu }}=T_{\text {downstream }}+T_{\text {upstream }},
$$

where $T_{\text {downstream }}$ and $T_{\text {upstream }}$ are time for sending data from OLT to ONU and ONU to OLT, respectively. From Eq. (2), $T_{\text {downstream }}$ can be defined as:

$$
T_{\text {downstream }}=T_{d}+T D_{\text {payload }},
$$

where $T_{d}$ is the propagation delay from OLT to ONU and $T D_{\text {payload }}$ is the frame length of the downstream payload, and $T_{\text {upstream }}$ can be defined as:

$$
T_{\text {upstream }}=T_{d}+T U_{\text {payload }},
$$

where $T_{d}$ is the propagation delay from ONU to OLT and $T U_{\text {payload }}$ is the frame length of the upstream payload.

Following [7], the standard of tactile internet in the optical access network has a latency time is about 0.5 milliseconds.

\subsection{Radio interface}

Radio interface is the WiFi connection, where the WiFi connection time refers to the time during the MU specified WiFi coverage area. IEEE 802.112016. [19] standardized a fine time measurement (FTM) protocol that enables a pair of $\mathrm{WiFi}$ connection to estimate the distance. The time-based distance measurements of WiFi-RTT [19] is intuitively based on the delay that the signal takes to travel from the sender to the receiver. An intuitive method to measure the propagation delay of a signal is the time of flight (ToF) [19] which is measured based on time differences at the sender and receiver. The $T o F$ equation can be defined as:

$$
\text { ToF }=\left(t_{4}-t_{1}\right)-\left(t_{3}-t_{2}\right)
$$

where $t_{1}$ is the current time that is the responder store in the memory, $t_{2}$ is the initiator records time, as soon as the incoming signal detected at the antenna, $t_{3}$ is the timestamp recorded at the initiator after receiving the FTM frame and send the acknowledge (ACK) frame at the initiator, $t_{4}$ is the responder calculate time for total round-trip time of the signal by subtracting $t_{1}$ from $t_{4}$.
Measuring the time of flight only once is not sufficient. Relatively small deviations from the realtime value result in a vast error in the distance estimate. For this reason, the procedure is repeated multiple times to reduce the impact of noise. The single FTM measurement consists of many FTMACK frame exchanges and the final value $T_{0} F^{*}$ [19] is the average over $N$ measurements. The $T o F^{*}$ can be defined as:

$$
\operatorname{ToF}^{*}=\frac{1}{N} \sum_{n=1}^{N}\left(\left(t_{4, n}-t_{1, n}\right)-\left(t_{3, n}-t_{2, n}\right)\right),
$$

where $n$ is the number of measurement times, from Eq. (6), the WiFi-RTT can defined as:

$$
T_{w r t t}=T_{p r o c}+T o F^{*},
$$

where $T_{\text {proc }}$ is the processing time for each packet and is set to $1 \mu \mathrm{s}[20]$.

The latency time in Radio interface is about 0.2 milliseconds [7]. The latency time of WiFi connection will depend on the number of MUs in the area and distance from the MU to the Access Point (AP) with the $2.4 \mathrm{GHz}$.

\subsection{Mobile unit interface}

The mobile unit is connected to the sensor or application for collecting the data, then the processed data is sent to the ONU. The latency time in MU is about 0.3 milliseconds [7]. The increased latency time will depend on the process running within each MU.

\section{Proposed system}

In this section, we introduce the FiWi to Dynamic Wavelength and Bandwidth Allocation (F-DWBA) algorithm to perform the latency budget of tactile internet system starting at the central office (CO) until the mobile unit (MU) at the end.

The tactile internet access networks require the strict of quality of service (QoS) data traffic. The accurate estimation of upcoming packets is necessary in order to perform efficiently for reducing the delay from the origin to the destination. Each ONU is assigned a set of wavelength and the window time in which a buffer can be sent the data. At the end of the transmission, each ONU will send a report message containing the useful information that will enable the central office to predict the future traffic and to determine the appropriate resources for each ONU. 


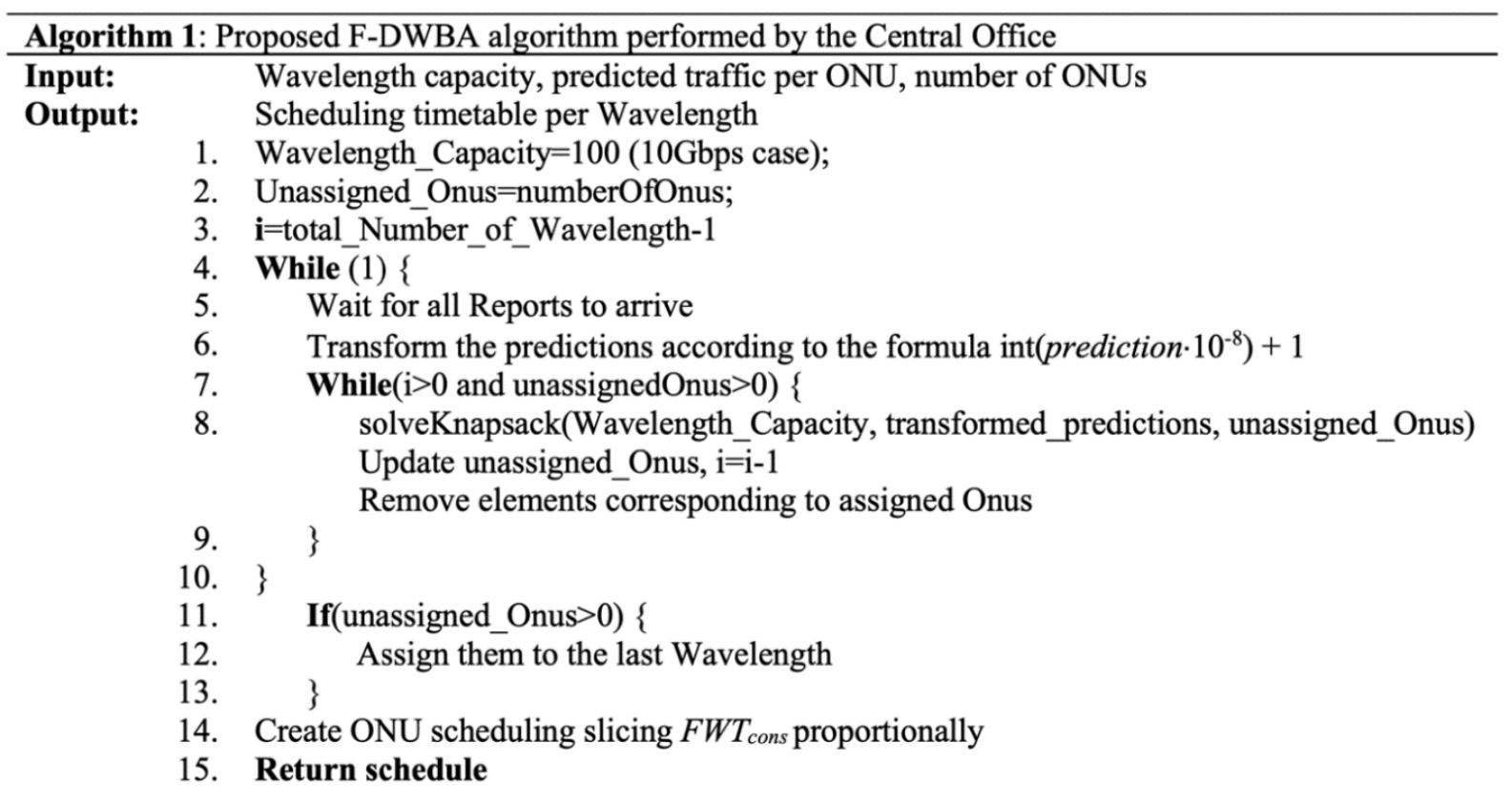

Figure. 7 Proposed FiWi to dynamic wavelength and bandwidth allocation (F-DWBA) algorithm

For the Dynamic Wavelength and Bandwidth Allocation (DWBA) algorithm [9], the researchers have improved the latency time from PON to ONU. The DWBA algorithm can limit the latency time between OLT to ONU by one millisecond, but the DWBA algorithm cannot support wireless connection. If the delay time between ONU and the MU has high latency, the DWBA algorithm dose not fully supports the tactile internet system.

With the F-DWBA algorithm, the end-to-end constraint $L_{\text {cons }}$ is embedded in the process of the PON system. The F-DWBA is considered an DBA $[5,21]$ algorithm. In the DBA algorithm, a packet arriving at the ONU waits for approximately half a transmission polling cycle, Tpoll_cycle [5]. This queuing delay, in conjunction with the round-trip time of $T_{r t t}$ and the processing time of $T_{p r o c}$, results in the following end-to-end packet latency, which $L_{\text {avg }}$ can be expressed as:

$$
L_{\text {avg }}=\frac{1}{2}\left(T_{\text {poll_cycle }}\right)+T_{\text {proc }}+T_{\text {rtt }},
$$

where $T_{\text {poll_cycle }}$ is the polling cycle time, $T_{\text {proc }}$ is the processing time for each packet, $T_{r t t}$ is the round-trip time. In the latency-constrained network, the $L_{\text {avg }}$ experienced by a packet should be less than that of the imposed constraint, $L_{\text {cons }}$.

$$
L_{\text {avg }} \leq L_{\text {cons }}
$$

Using Eq. (8) and Eq. (9), for latency can be written as follows:

$$
T_{\text {cycle }} \leq 2\left(L_{\text {cons }}-T_{\text {proc }}-T_{\text {rtt }}\right)
$$

Therefore, it follows that the maximum polling cycle time $T_{\text {maxpoll }}$ is given by:

$$
T_{\text {maxpoll }}=2\left(L_{\text {cons }}-T_{\text {proc }}-T_{\text {rtt }}\right)
$$

One millisecond latency is required to support the tactile internet. We consider that the constraint is a maximum of 0.5 millisecond round-trip time for the proposed network. In order to achieve the delay time constraint at 0.5 milliseconds for real-time applications, the fiber-wireless time $\left(F W T_{\text {cons }}\right)$ can be expressed as:

$$
F W T_{\text {cons }}=0.95 \cdot 2\left(L_{\text {cons }}-T_{\text {proc }}-T_{\text {rtt fiwi }}\right),
$$

where $L_{\text {cons }}$ is set to 0.5 milliseconds. $T_{\text {proc }}$ is the processing time for each packet and is set to $1 \mu \mathrm{s}$ [20]. $T_{r t t \text { fiwi }}$ is the round trip time of the furthest ONU plus the round trip time of WiFi as:

$$
T_{\text {rtt fiwi }}=T_{\text {rtt onu }}+T_{w r t t},
$$

where $T_{\text {rtt onu }}$ is the round trip time of the furthest ONU that should be a maximum round-trip time at less than 0.5 millisecond for the network. $T_{w r t t}$ is the round trip time of $\mathrm{WiFi}$. The guard band factor is set to 0.95 . In the simulation, we consider the Tactile 
Internet (TI) and Non-Tactile Internet (Non-TI), which TI is considered to follow $F W T_{\text {cons }}$ process and Non-TI is the standard process.

In our interest, we focus on the end-to-end delay. The tactile internet functionality must support more mobile unit. Therefore, we consider the importance of delay time at radio interface and MU interfac. The proposed FiWi to Dynamic Wavelength and Bandwidth Allocation (F-DWBA) algorithm is presented in Fig. 7.

From algorithm 1 in Fig. 7, the proposed FDWBA algorithm can divided into two steps, consisting of dynamic wavelength and bandwidth allocation and traffic flow management. From line 1 to line 13 are dynamic wavelength and bandwidth allocation step. From line 1 to line 3, the OLT will be making the schedule, and the other is the assignment of wavelengths, in this case of wavelength capacity at 100 for the case of 10Gbps used. In the phase generation schedule of line 4 to line 10, we arrange the ONUs in ascending order based on round-trip time (RTT). If the ONU has a TI application, that ONU will come first. If two ONUs have the same RTT as the TI application, then the ONU with the shortest execution time comes first in the scheduling sequence. When the create phases are complete, we will check the availability of wavelengths. If the wavelength is available, we will assign the available wavelengths to the ONU at the top of the sorted list. We will allocate the wavelength to the next available ONU in the same manner, as line 11. Line 15 shows the traffic flow management step, we will create ONU with the tactile internet applications scheduling by using Eq. (12), $F W T_{\text {cons }}$. After the allocated is complete, the ONU can send data to the OLT during the allocated interval with a report message for the next bandwidth request.

\section{Simulation setup and results}

In this section, the experiments with the upstream traffic are performed through computer simulations to verify the proposed algorithm. The Huawei OLT and ONU models are widely used with a distributed G-PON architecture [22], which are positioned as the next generation OLT and ONU for the NextGeneration Passive Optical Network (NG-PON). The upstream wavelength is capable to provide up to 10Gbps, while the downstream can provide up to 10Gbps. The parameters used in the experiments are based on Huawei models. For the G-PON fiber network, the parameters of fiber optic cable are followed the ITU-T G.652 standard [17]. For the packet size of TI and Non-TI, the Transmission
Table 1. Simulation parameters

\begin{tabular}{|c|c|}
\hline Simulation Parameters & Values \\
\hline Number of ONUs & 4 \\
\hline Distance OLT-ONU & $1,2,3, \ldots, 10 \mathrm{~km}$ \\
\hline Number of TI MU & 2 \\
\hline Number of User & 4 \\
\hline Latency Constraint & $500 \mathrm{us}$ \\
\hline Number of Wavelengths & 1 \\
\hline Link Capacity & $10 \mathrm{Gbps}$ \\
\hline ONU buffer & $1 \mathrm{MB}$ \\
\hline TI packet length & $64 \mathrm{bytes}$ \\
\hline Non TI packet length & $512 \mathrm{bytes}$ \\
\hline WiFi frequency band & $2.4 \mathrm{GHz}$ \\
\hline $\mathrm{T}_{\text {wrtt }}$ & $100 \mathrm{us}, 200 \mathrm{us}$ \\
\hline
\end{tabular}

700 us round-trip delay limit

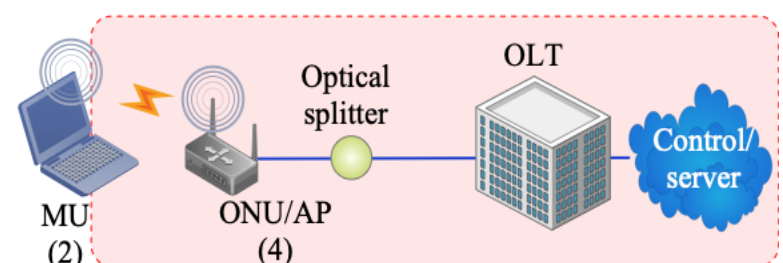

Figure. 8 Experiment setup for fiber wireless access

Control Protocol (TCP) and User Datagram Protocol (UDP) standard are applied with the frequency band of WiFi applications at $2.4 \mathrm{GHz}$ [23]. A simulator was built parameters as shown in Table 1 . The guard band factor is set to 0.95 and the time constraint is $500 \mu$ s.

Experiments are divided into two sections. The first section, we focus on the fiber wireless access network. The connection from radio interface of MU to the cloud server and round-trip delay is limited at $700 \mu \mathrm{s}$. The second section, we focus on the overall tactile internet system. The round-trip delay is onemillisecond limit.

\subsection{Fiber wireless access network}

The connection from MU radio interface to the cloud server is limited the round-trip delay at $700 \mu$ s. We perform this experiment following in Fig. 8 including with 4 ONUs and 2 APs connected to 2 MUs at the end of system.

Fig. 9 shows the round-trip delay for MU in the distance of fiber optic plus with WiFi from OLT to MU, where $T_{w r t t}=100 \mu \mathrm{s}$. Simulation results at $10 \mathrm{~km}$ show that the round-trip delay of GPON with non-TI is $750 \mu \mathrm{s}$, while the DWBA [9] has a round-trip delay of $700 \mu$ s. The proposed F-DWBA algorithm has a round-trip delay of $400 \mu$ s, where the round-trip delay for TI is limited at $700 \mu \mathrm{s}$.

The round-trip delay of GPON with Non-Ti, DWBA with TI and propsed F-DWBA from OLT to 


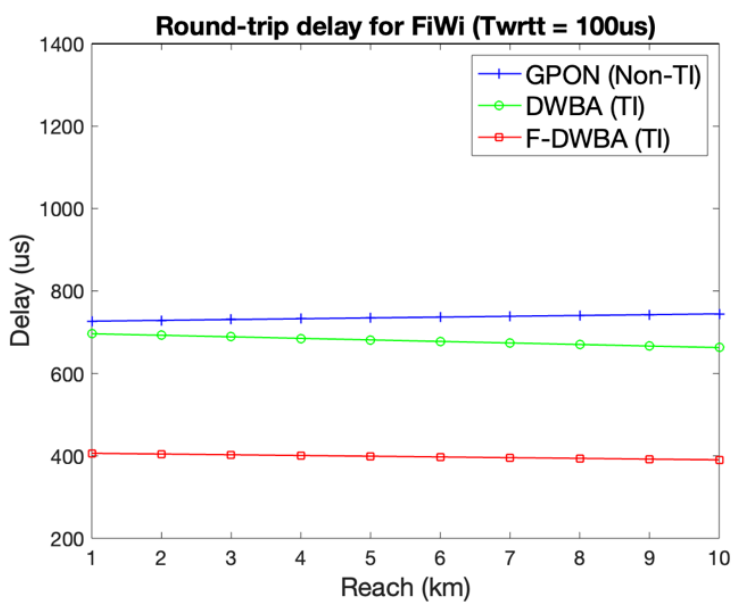

Figure. 9 Round-trip delay of MU at $T_{w r t t}=100 \mu \mathrm{s}$

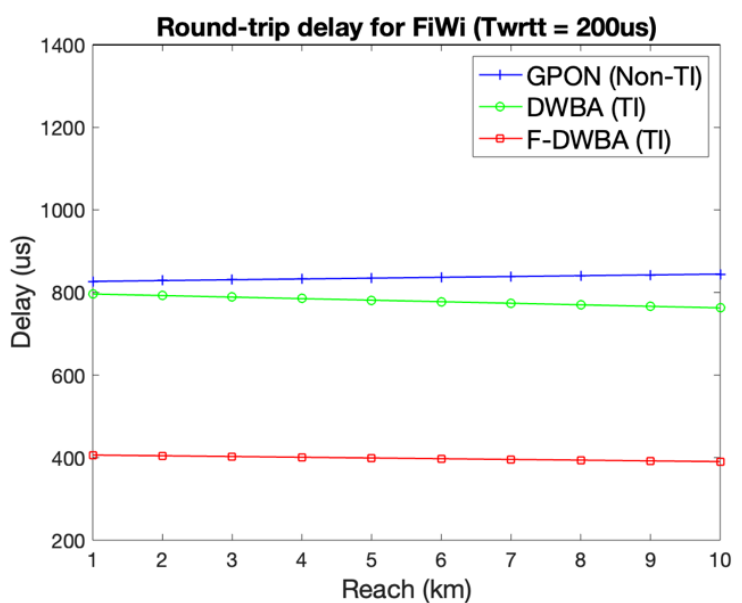

Figure. 10 Round-trip delay of MU at $T_{w r t t}=200 \mu$ s.

ONU for MU at $T_{w r t t}=200 \mu$ s are shown in Fig. 10 . The guard band factor is set to 0.95 for reducing the polling cycle time. The required time constraint is fixed at $500 \mu \mathrm{s}$. For the distance at $10 \mathrm{~km}$, the roundtrip delay of GPON with non-TI is $820 \mu$ s and of DWBA [9] with TI is $800 \mu$ s. For the proposed FDWBA algorithm with $\mathrm{TI}$, the round-trip delay is $400 \mu \mathrm{s}$, where the round-trip delay for TI is limited at $700 \mu \mathrm{s}$.

\subsection{Tactile internet system and throughput}

In this section, we focus on the overall tactile internet system, which the target of round-trip delay is limited at one-millisecond. We perform this experiment including with 4 ONUs and 2 APs connected to $2 \mathrm{MUs}$ at the end of system following in Fig. 11.

The experimental results show the round-trip delay of TI at $T_{w r t t}=100 \mu \mathrm{s}, 200 \mu \mathrm{s}$ with onemillisecond limit in Fig. 12 and Fig. 13, respectively. Fig. 12 shows the simulation results at $10 \mathrm{~km}$, that the

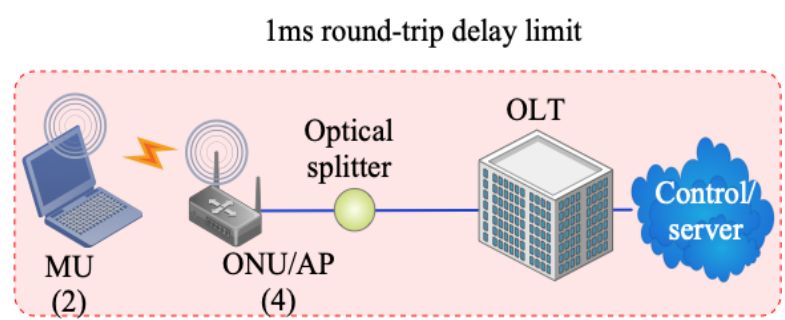

Figure. 11 Experiment setup for tactile internet system and throughput.

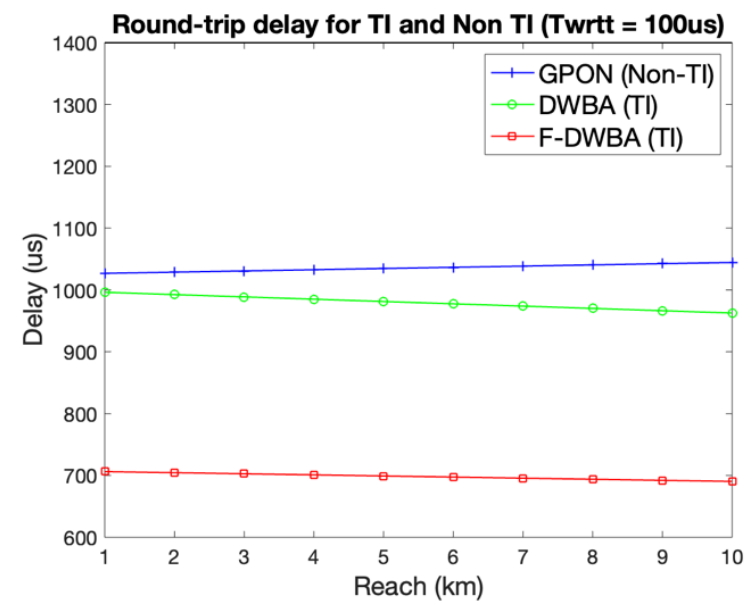

Figure. 12 Round-trip delay of TI at $T_{w r t t}=100 \mu \mathrm{s}$

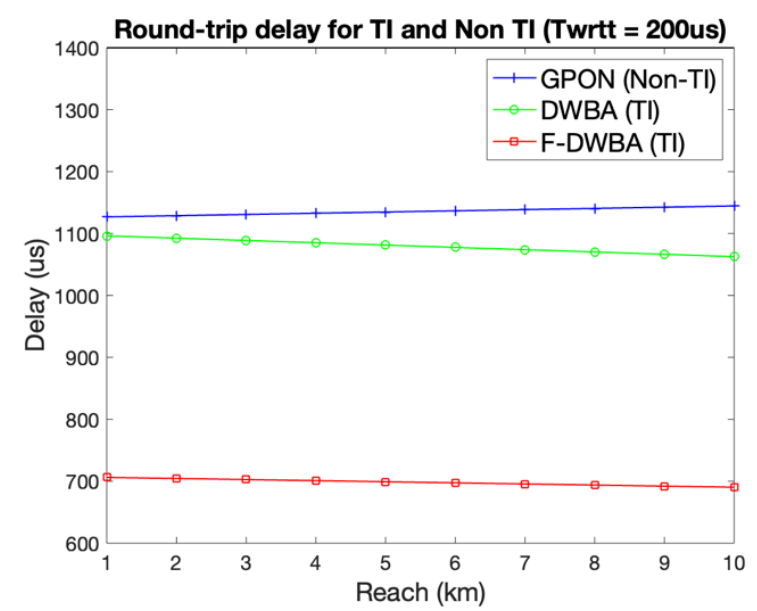

Figure. 13 Round-trip delay of TI at $T_{w r t t}=200 \mu \mathrm{s}$

round-trip delay of GPON with non-TI is $1020 \mu \mathrm{s}$, while the DWBA [9] has a round-trip delay of $990 \mu \mathrm{s}$. The proposed F-DWBA algorithm has a round-trip delay of $700 \mu \mathrm{s}$.

Simulation results of the round-trip delay of OLT to ONU for MU at $T_{w r t t}=200 \mu$ s are shown in Fig 13. At $10 \mathrm{~km}$, the GPON non-TI has a round-trip delay at $1150 \mu \mathrm{s}$. The DWBA [9] has a round-trip delay of $1050 \mu \mathrm{s}$. For the proposed F-DWBA algorithm, a round-trip delay is of $700 \mu$ s. It is seen that the proposed F-DWBA algorithm can allocate 


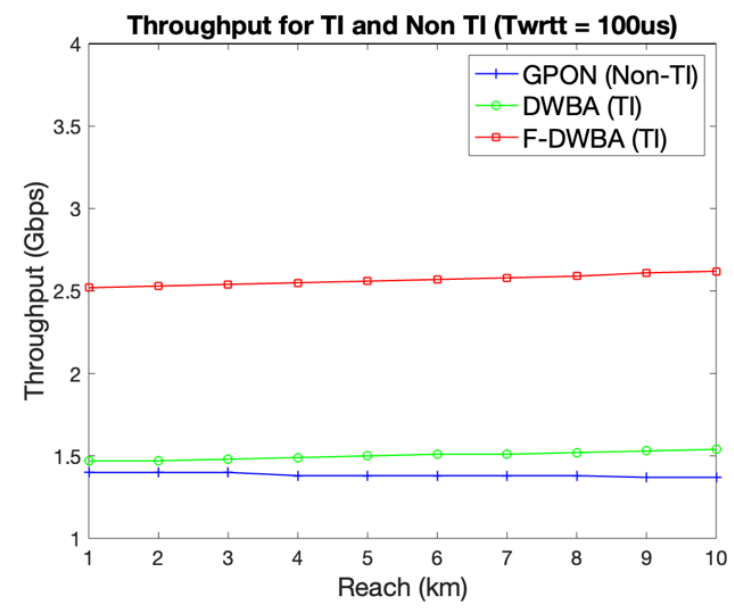

Figure. 14 Throughput for TI at $T_{w r t t}=100 \mu \mathrm{s}$

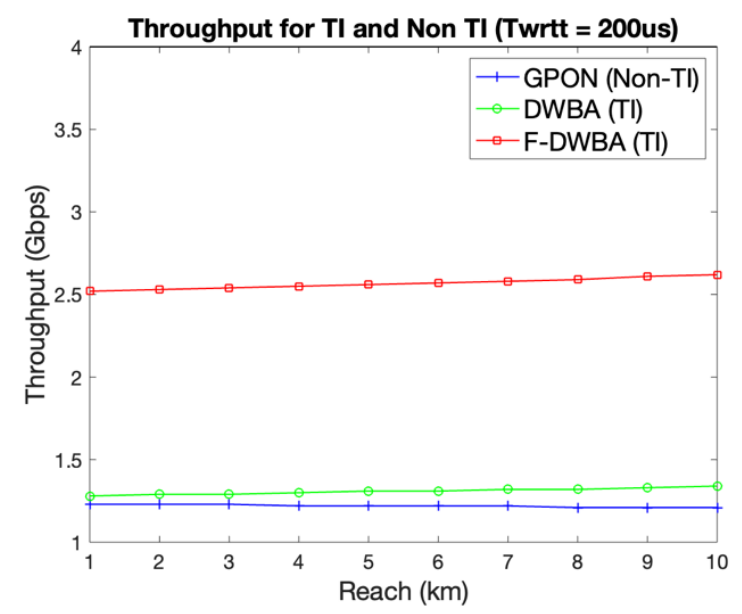

Figure. 15 Throughput for TI at $T_{w r t t}=200 \mu \mathrm{s}$

the best round-trip delay for the tactile internet system compared with GPON with Non-Ti and DWBA [9].

Fig. 14 presents the ONU throughput for the MU at $T_{w r t t}=100 \mu \mathrm{s}$ at the range of $10 \mathrm{~km}$. The proposed F-DWBA with TI can achieve a throughput of 2.5Gbps. The DWBA [9] and GPON are approximately the same at $1.5 \mathrm{Gbps}$. It is noticed that the proposed F-DWBA algorithm can deliver better throughput in comparison with GPON with Non-TI and DWBA [9], which can support the TI applications.

Fig. 15 shows the ONU throughput for the MU at $T_{w r t t}=200 \mu$ s at the range of $10 \mathrm{~km}$. The proposed F-DWBA with TI has a throughput of $2.5 \mathrm{Gbps}$. Meanwhile, the DWBA and GPON with TI are approximately of $1.3 \mathrm{Gbps}$. It is found that the proposed F-DWBA can deliver throughput better than GPON with Non-TI and DWBA [9] and the proposed F-DWBA can still support the TI applications at $T_{w r t t}=200 \mu \mathrm{s}$.

Finally, Fig. 16 shows the throughput

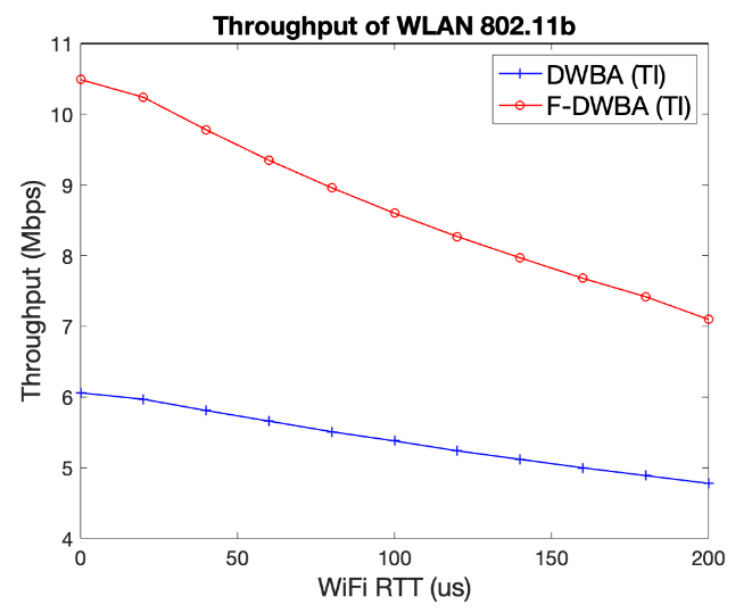

Figure. 16 Throughput of WLAN 802.11b

performance of WLAN 802.11b with the different of WiFi round trip time (WiFi-RTT) delay. Without delay, the proposed F-DWBA with TI has a maximum throughput of $10.5 \mathrm{Mbps}$, while the DWBA with TI has a maximum throughput of $6 \mathrm{Mbps}$. For $100 \mu$ s delay, the proposed F-DWBA with TI has a maximum throughput of $8.9 \mathrm{Mbps}$ and the DWBA [9] with TI has a maximum throughput of 5.5Mbps. And the proposed F-DWBA with TI has a maximum throughput of $7 \mathrm{Mbps}$ and the DWBA [9] with TI has a maximum throughput of $4.8 \mathrm{Mbps}$, where the delay is nearby $200 \mu$ s.

\section{Conclusion}

In this paper, we have introduced the proposed FDWBA algorithm for low-latency FiWi enhancement with tactile internet on the PON and POLAN. Meanwhile, PONs can satisfy the stringent TI requirements as the low latency and high reliability for both IoT and IIoT applications.

Experimental simulations have proved that the round-trip delay can support the tactile traffic. The constraint of the required time at $500 \mu$ s can help to calculate the round-trip delay in the proposed system. The first section of experiment, we have focused on fiber wireless access network. It is noted that the TI packet of round-trip delay is lower than one millisecond limit. The proposed F-DWBA algorithm has more better round-trip delay than G-PON standard about 46 percentage and better than DWBA algorithm about 42 percentage at $T_{w r t t}=100 \mu \mathrm{s}$. For $T_{w r t t}=200 \mu \mathrm{s}$, the proposed F-DWBA algorithm has a better round-trip delay than G-PON standard and DWBA algorithm about 51 percentage and 50 percentage, respectively.

The second section of experiment, we have focused on the overall tactile internet system. The TI packet round-trip delay is lower than one-millisecond 
limit. At $T_{w r t t}=100 \mu \mathrm{s}$, the proposed F-DWBA algorithm has a better round-trip delay than G-PON with Non-TI about 31 percentage and better than DWBA about 29 percentage. For $T_{w r t t}=200 \mu \mathrm{s}$, the proposed F-DWBA algorithm has more better roundtrip delay than G-PON standard and DWBA algorithm about 40 percentage and 33 percentage, respectively.

The proposed F-DWBA algorithm has an ONU throughput of $2.5 \mathrm{Gbps}$ at $T_{w r t t}=100 \mu \mathrm{s}$ and $200 \mu \mathrm{s}$. The throughput performance of WLAN 802.11b for the proposed F-DWBA algorithm has a maximum throughput of $10.5 \mathrm{Mbps}$ without the delay. When the delay is nearby $200 \mu \mathrm{s}$, the proposed F-DWBA algorithm has a maximum throughput of $7 \mathrm{Mbps}$. Therefore, the proposed F-DWBA algorithm can lead to a new generation for PON and POLAN which are capable of supporting IoT and IIoT applications.

In future work, the $5 \mathrm{G}$ networks and industrial IoT with smart factories and Industry 4.0 are being interested. Based on the Industry 4.0, the organizations need the fast, reliable, and secure wireless connection solution in order to achieve the high performance with the low latency of communication.

\section{Conflicts of interest}

The authors declare no conflict of interest.

\section{Author contributions}

Conceptualization, Jirasak Ponchua and Suchada Sitjongsataporn; methodology, Jirasak Ponchua and Suchada Sitjongsataporn; validation, Jirasak Ponchua and Suchada Sitjongsataporn; writing — original draft preparation, Jirasak Ponchua; writing - review and editing, Jirasak Ponchua; visualization, Suchada Sitjongsataporn; supervision, Suchada Sitjongsataporn.

\section{Acknowledgments}

The authors would like to extend their sincere appreciation to Assist. Prof. Dr. Theerayod Wiangtong at King Mongkut's Institute of Technology Ladkrabang for his support and suggestion in this research.

\section{References}

[1] ITU-T, "Overview of the Internet of thing", Y.2026, 2012.

[2] S. Prongnuch, T. Wiangtong, and S. Sitjongsataporn, "Qualitative Precipitation Estimation from Satellite Data Based on Distributed Domain-Specific Architecture",
Modelling and Simulation in Engineering (MSE), Vol. 2021, 2021.

[3] D. Serpanos and M. Wolf, "Internet-of-Things (IoT) Systems", Springer International Publishing, 2018.

[4] ITU-T, "Overview of smart manufacturing in the context of the industrial Internet of things", Y.4003, 2018.

[5] ITU-T, "Gigabit-capable passive optical networks (GPON)", G.984 Series, 2019.

[6] G. Simon, A. Ankouri, L. Neto, P. Chanclou, and D. Kurz, "Ftth and Optical LAN Synergy Enabled by Virtual OLT for Home, Office and Campus", In: Proc. of International Conference System Modeling and Advancement in Research Trends (SMART), pp. 1-3, 2019.

[7] ITU-T, "The Tactile Internet", ITU-T Technology Watch Report, 2014.

[8] M. Maier, "Fiber-Wireless (FiWi) Broadband Access Networks in an Age of Convergence: Past, Present, and Future", Advances in Optics, Vol. 2014, article ID 945364, 2014.

[9] G. Tziroglou, A. Valkanis, C. Kyriakopoulos, G. Papadimitriou, and P. Nicopolitidis, "On the use of prediction in Passive Optical LANs for healthcare latency-stringent applications", In: Proc. of IEEE EUROCON, pp. 1-6, 2019.

[10] R. Sriram and A. Sheth, "Internet of Things Perspectives", IT Professional, Vol. 17, No. 3, pp. 60-63, 2015.

[11] B. Bajic, A. Rikalovic, N. Suzic, and V. Piuri, "Industry 4.0 Implementation Challenges and Opportunities: A Managerial Perspective" IEEE Systems Journal, Vol. 15, No. 1, pp. 546-559, 2021.

[12] H. Yang, S. Kumara, S. T. S. Bukkapatnam, and F. Tsung, "The Internet of Things for smart manufacturing: A review", IISE Trans., Vol. 51, No. 11, pp. 1190-1216", 2019.

[13] R. Jirachariyakool, N. S. Ium, and S. Lerkvaranyu, "Design and Implement of GPON-FTTH network for residential condominium", In: Proc. of JCSSE, pp. 1-5, 2017.

[14] A. Samir, J. Ratkoceri, and B. Batageli, "MultiCore Optical Fiber in a Passive Optical Local Area Network", In: Proc. of ITCE, pp. 77-82, 2018.

[15] G. P. Fettweis, "The Tactile Internet: Application and Challenges", IEEE Vehicular Technology Magazine, 2014.

[16] J. Kleinberg and E. Tardos, Algorithm Design. Harlow: Pearson Education, 2014.

[17] ITU-T, "G.652: Characteristics of a single-mode optical fibre and cable", G.652 series, 2016. 
[18] K. Lee, J. Lee, Y. Yi, I. Rhee, and S. Chong, "Mobile data offloading: How much can WiFi deliver?", IEEE/ACM Trans. Netw., Vol. 21, No. 2, pp. 536-550, 2013.

[19] IEEE, "Part 11: Wireless LAN Medium Access Control (MAC) and Physical Layer (PHY) Specifications", IEEE Standard 802.11-2016, 2016.

[20] X. Hu, X. Chen, Z. Zhang, and J. Bei, "Dynamic Wavelength and Bandwidth Allocation in Flexible TWDM Optical Access Network", IEEE Communications Letters Trans., Vol. 18, No. 12, pp. 2113- 2116, 2014.

[21] E. Wong, M. P. I. Dias and L. Ruan, "Predictive Resource Allocation for Tactile Internet Capable Passive Optical LANs", Journal of Lightwave Technology, Vol. 35, No. 13, pp. 2629-2641, 2017.

[22] Huawei, "SmartAX Optical Line Terminal", Huawei SmartAX OLT Datasheet, 2021.

[23] ITU-T, "Internet protocol data communication service", Y.1540, 2019. 